\title{
METÁSTASIS COROIDEAS DE CARCINOMA DE MAMA. DIAGNÓSTICO Y SEGUIMIENTO MEDIANTE TOMOGRAFÍA DE COHERENCIA ÓPTICA Y ANGIOFLUORESCEINGRAFÍA Y AUTOFLUORESCENCIA CON HRA-II (HEIDELBERG RETINA ANGIOGRAPH)
}

\author{
CHOROIDAL METASTASIS FROM A BREAST CARCINOMA. \\ DIAGNOSIS AND FOLLOW-UP WITH OPTICAL COHERENCE \\ TOMOGRAPHY AND FLUORESCEIN ANGIOGRAPHY AND \\ AUTOFLUORESCENCE WITH HRA-II (HEIDELBERG RETINA \\ ANGIOGRAPH)
}

PÉREZ-ÁLVAREZ MJ ${ }^{1}$, ARRIOLA-VILLALOBOS P ${ }^{1}$, RECHE-FRUTOS J ${ }^{1}$, GARCÍA-SÁNCHEZ J²

\section{RESUMEN}

Caso clínico: Mujer de 69 años que desarrolló metástasis coroideas unilaterales a partir de un carcinoma de mama, tratado con cirugía y quimioterapia. Evaluamos la resolución del desprendimiento seroso retiniano mediante tomografía de coherencia óptica (OCT) y angiofluoresceingrafía (AFG) tras quimioterapia y radioterapia externa, y la posterior recidiva en el nervio óptico.

Discusión: La OCT resulta útil en el diagnóstico de cambios secundarios retinianos, aunque su valor es más limitado en la localización inicial de las lesiones. Sin embargo, es de gran utilidad en el seguimiento. La autofluorescencia permite detectar actividad exudativa tumoral, incluso sin cambios evidentes en AFG u OCT.

Palabras clave: Metástasis coroideas, OCT, AFG, HRA-II.

\begin{abstract}
Clinical case: A 69-year-old woman developed choroidal metastasis from a breast carcinoma 2 years after the initial diagnosis, surgery and chemotherapy. After treatment with palliative chemotherapy and external radiotherapy, we used fluorescein angiography (FA) and optical coherence tomography (OCT) to evaluate the resolution of the serous retinal detachment, as well as a later relapse in the optic nerve.

Discussion: OCT is useful in the follow-up of choroidal metastasis after treatment. However, OCT imaging is limited by the initial choroidal location of metastasis. The autofluorescence can detect exudative tumoral activity even without obvious changes in OCT or FA (Arch Soc Esp Oftalmol 2009; 84: 267-270).
\end{abstract}

Key words: Choroidal metastasis, Fluorescein Angiography, HRA-II, OCT.

\footnotetext{
Recibido: 21/12/07. Aceptado: 12/5/09.

Hospital Clínico San Carlos. Unidad de Retina. Madrid. España.

${ }^{1}$ Licenciado en Medicina.

2 Doctor en Medicina.

Correspondencia:

María Jesús Pérez Álvarez

$\mathrm{P}^{\circ}$ de los Pontones, $5,4 .^{\circ}$ Dcha.

28005 Madrid

España

E-mail: mjperezalv@ hotmail.com
} 


\section{INTRODUCCIÓN}

Las metástasis coroideas son el tumor ocular más frecuente. Los tumores primarios más frecuentes en las metástasis coroideas se localizan en el pulmón y la mama (1).

La radioterapia es generalmente el tratamiento de elección. Debe asociarse quimioterapia cuando se demuestran otras metástasis sistémicas. El 53\% de los pacientes mejoraron su agudeza visual y el $34 \%$ no mostraron cambios. En el $83 \%$ de los casos se consigue control local del tumor con escasos efectos secundarios (2).

\section{CASO CLÍNICO}

Presentamos una mujer de 69 años, diagnosticada de carcinoma ductal infiltrante de mama derecha no metastático en 2004. Fue tratada mediante mastectomía radical con linfadenectomía y recibió quimioterapia coadyuvante FAC (adriamicina $80 \mathrm{mg}$, 5-fluoracilo $800 \mathrm{mg}$, genoxal $800 \mathrm{mg}$ ) 6 ciclos con un dosis total de $480 \mathrm{mg}$, durante 5 meses con buena tolerancia. En junio 2006, se realizó cirugía de reconstrucción con prótesis expansora.

En agosto 2006 presentó derrame pleural derecho metastásico, confirmado mediante biopsia bronquial y lesiones blásticas en articulación esternoclavicular.

Durante el estudio de extensión presentó disminución brusca de agudeza visual (AV) en el ojo dere-

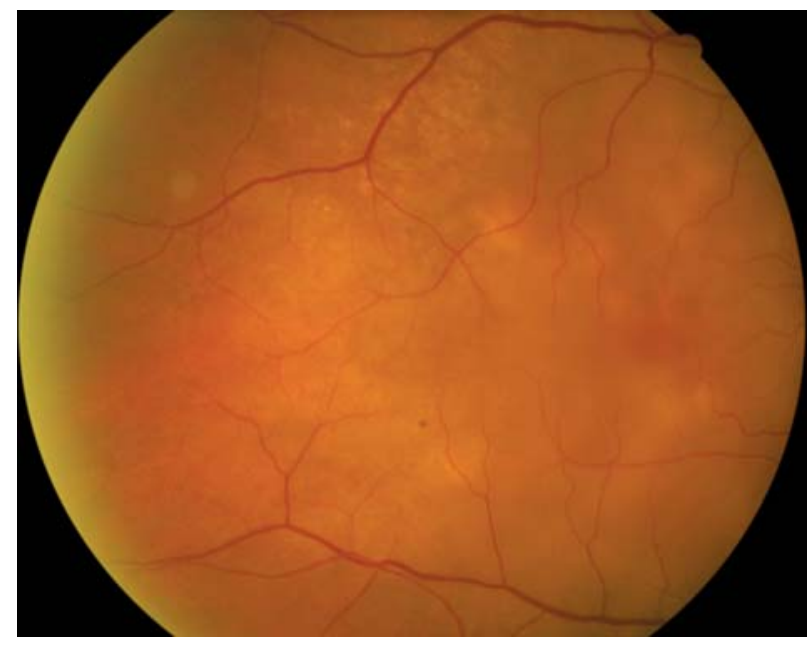

Fig. 1: Retinografía de ojo derecho que muestra desprendimiento seroso. cho (OD). La exploración demostró: AV ojo derecho (OD) 0,16 y ojo izquierdo (OI) 0,8. Biomicroscopía anterior y tonometría normales. En la funduscopía se apreció desprendimiento seroso foveal sin otros signos de degeneración macular asociada a la edad (fig. 1), siendo el ojo contralateral normal. La angiofluoresceingrafía (AFG) con HRA-II (Heidelberg Retina Angiograph) mostró desprendimiento del epitelio pigmentario retiniano (DEP) circunscrito sin signos de membrana neovascular coroidea (MNVC) (fig. 2). En la angiografía con verde indocianina (ICG) tampoco se objetivó neovascularización. La tomografía de coherencia óptica (OCT) permitió apreciar DEP foveal, desprendimiento seroso de la retina neurosensorial asociado y engrosamiento macular de 680 micras (fig. 3).

Durante los siguientes 10 días, el DEP macular aumentó de tamaño y presentó un desprendimiento seroso de la hemirretina inferior con disminución de la AV hasta movimiento de manos. Ante la sospecha de enfermedad metastásica solicitamos resonancia magnética nuclear cerebral, que mostró el desprendimiento de retina sin otras lesiones asociadas.

Se catalogó a la paciente como carcinoma de mama estadio IV e instauró quimioterapia paliativa y radioterapia externa. Un mes después de finalizada la radioterapia y tras 12 ciclos de quimioterapia,

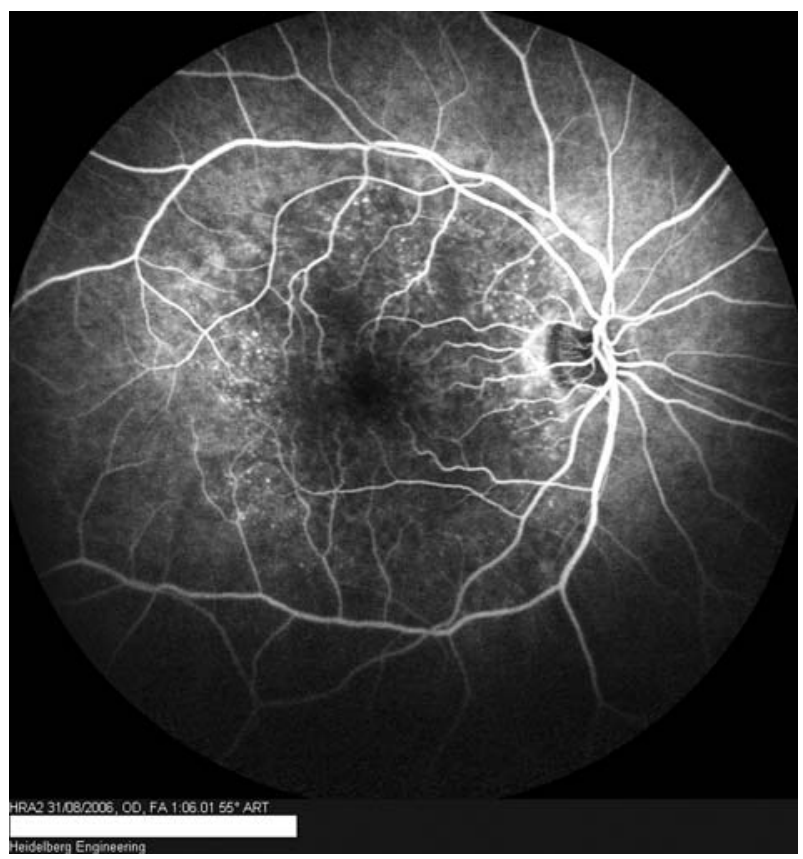

Fig. 2: Hiperfluorescencia en angiografía fluoresceínica $(A F G)$ de ojo derecho. 


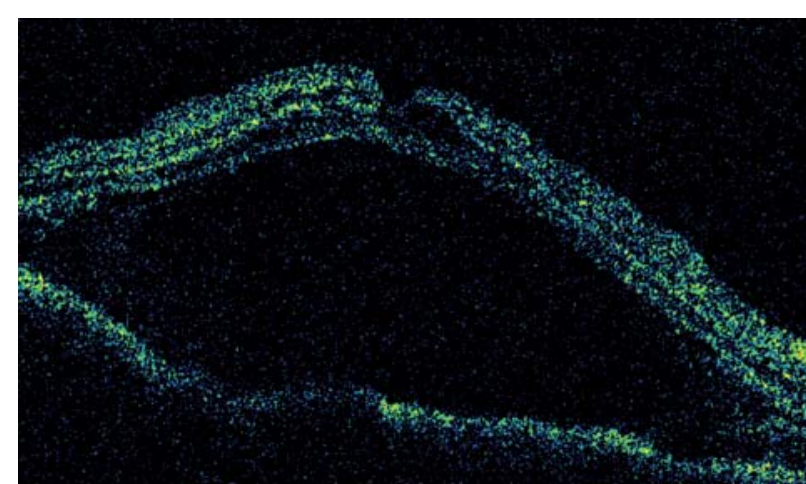

Fig. 3: Tomografía de coherencia óptica (OCT) en el que se aprecia gran desestructuración de la arquitectura retiniana.

la AV mejoró a 0,1 , el desprendimiento seroso se resolvió con atrofia del epitelio pigmentario de la retina (EPR) y el grosor foveal disminuyó hasta 250 micras (fig. 4).

Dos meses después presentó hemorragia en astilla y edema peripapilar sin desprendimiento seroso de otras zonas retina (fig. 5). La autofluorescencia y la imagen con infrarrojos (fig. 6) mediante HRA-II, mostraron muy leve hiperfluorescencia. Se valoró la posibilidad de nueva metástasis coroidea con afectación del nervio óptico, sin embargo no se modificó el tratamiento sistémico porque se oncología consideró que existe un beneficio clínico de la enfermedad sin progresión con la quimioterapia. La paciente no ha presentado nuevas metástasis viscerales en un año y medio de seguimiento.

\section{DISCUSIÓN}

Las metástasis coroideas son el tumor ocular más frecuente. Los tumores primarios más frecuentes en

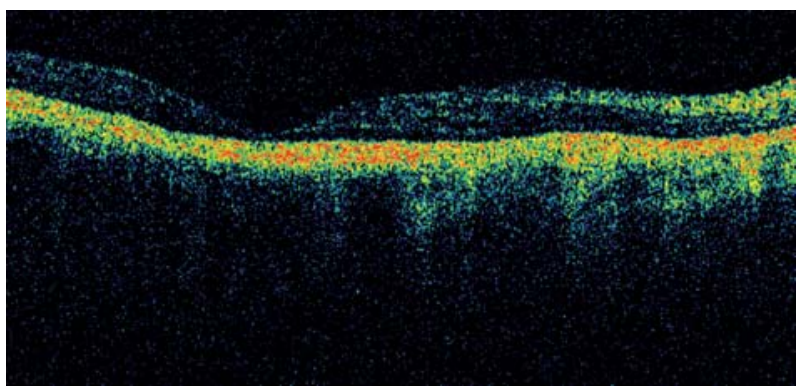

Fig. 4: Tomografía de coherencia óptica (OCT) que muestra resolución del desprendimiento seroso y normal grosor macular.

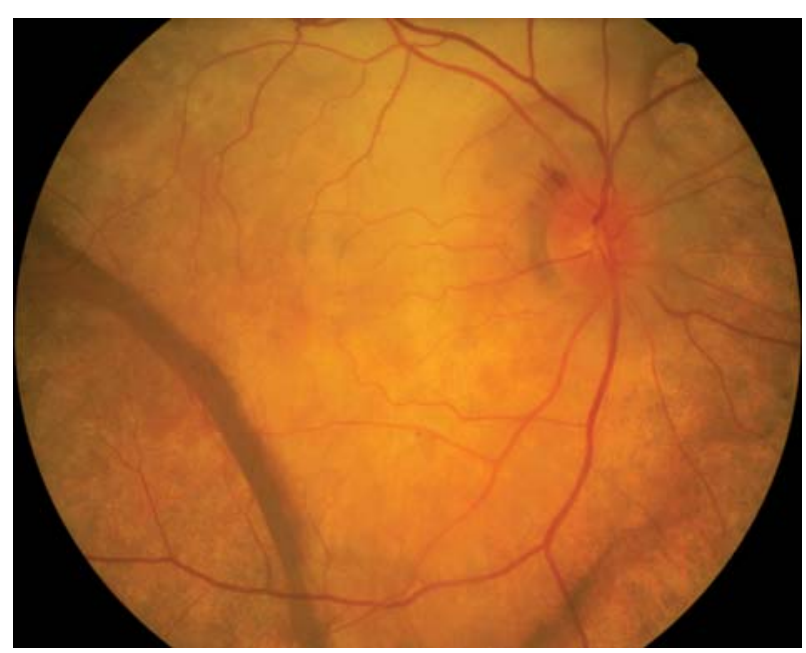

Fig. 5: Retinografía en la que se objetiva hemorragia en llama peripapilar superior.

las metástasis coroideas se localizan en el pulmón y la mama (1).

La radioterapia es generalmente el tratamiento de elección. Debe asociarse quimioterapia cuando se demuestran otras metástasis sistémicas. El 53\% de los pacientes mejoraron su agudeza visual y el $34 \%$ no mostraron cambios. En el $83 \%$ de los casos se consigue control local del tumor con escasos efectos secundarios (2).

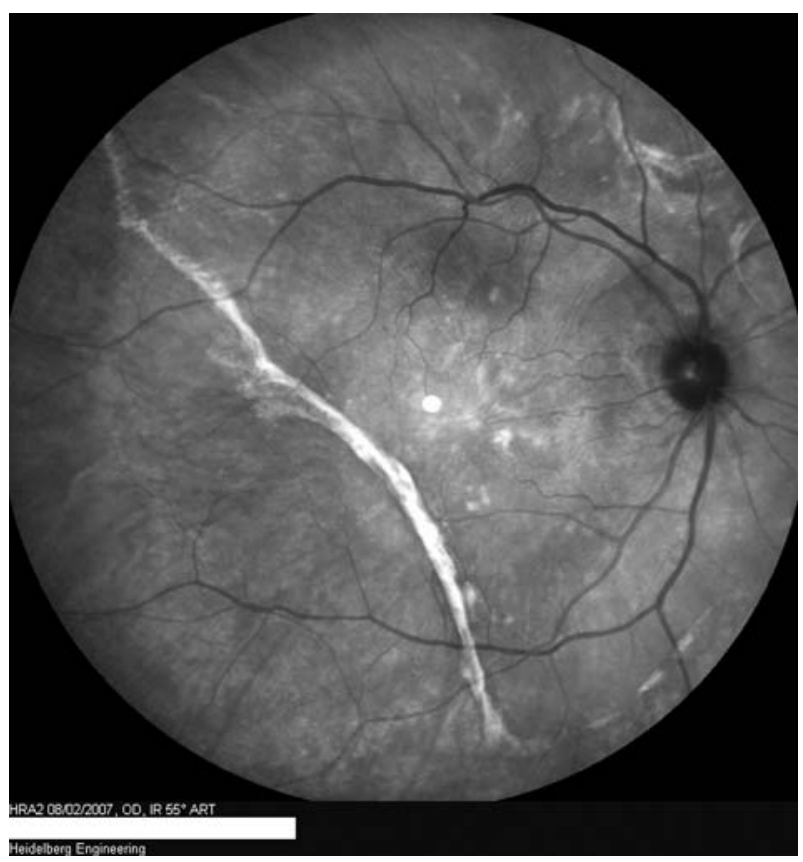

Fig. 6: Autofluorescencia con infrarrojos en HRA-II. 
La OCT ha demostrado su utilidad en el diagnóstico de los cambios secundarios en el EPR y en la retina, pero no permite el diagnóstico exacto de la localización coroidea de la metástasis debido a la dispersión a nivel del EPR. Sin embargo, resulta de gran utilidad, como ilustra nuestro caso, en el seguimiento de las lesiones asociadas y su evolución con el tratamiento (3). Nuestra paciente no debutó con lesiones coroideas identificables como masas cremosas, sino con desprendimiento seroso como complicación retiniana de la metástasis, por tanto no fue posible identificar la metástasis coroidea por funduscopía, AFG ni OCT. Sin embargo, los antecedentes personales y la existencia de otras metástasis sistémicas, sugerían la posibilidad de metástasis oculares. La OCT resultó de gran utilidad en el seguimiento como demuestra la normalización de la arquitectura retiniana tras el tratamiento oncológico.

La aparición de hemorragia peripapilar en astilla tras la resolución del desprendimiento seroso, sugiere la posibilidad de una metástasis a nivel del nervio óptico. Las metástasis en el disco suelen generar engrosamiento difuso del mismo en el 84\% de los casos, aunque pueden asociar hemorragias en el $42 \%$ (4). En nuestro caso nos planteamos diagnóstico diferencial con otras causas de hemorragias y edema nervio óptico, como retinopatía por radia- ción que, fue descartada por la ausencia de áreas de no perfusión en AFG y la aparición precoz del cuadro. Podemos concluir que nuevas técnicas de imagen como la autofluorescencia y la imagen con infrarrojos mediante HRA-II, constituyen un método no invasivo, que permite apreciar el componente seroso, y por tanto evaluar la actividad tumoral (5).

\section{BIBLIOGRAFÍA}

1. Barak A, Neudorfer M, Heilweil G, Merimsky O, Lowenstein A, Inbar M, et al. Decreased prevalence of asymptomatic choroidal metastasis in disseminated breast and lung cancer: argument against screening. $\mathrm{Br} J$ Ophthalmol 2007; 91: 74-75.

2. Bottke D, Wiegel T, Kreusel KM, Bornfeld N, Schaller G, Hinkelbein W. Radiotherapy of choroidal metastases in patients with disseminated cancer. Onkologie 2000; 23: 572-575.

3. Arevalo JF, Fernandez CF, Garcia RA. Optical coherence tomography characteristics of choroidal metastasis. Ophthalmology 2005; 112: 1612-1619.

4. Shields JA, Shields CL, Singh AD. Metastatic neoplasms in the optic disc: the 1999 Bjerrum Lecture: part 2. Arch Ophthalmol 2000; 118: 217-224.

5. Jork J, Bindewald A, Dithmar S, Holz, F. Digital simultaneous fluorescein and indocyanine green angiography, autofluorescence, and red-free imaging with a solid-state laser-based confocal scanning laser ophthalmoscope. Retina 2005; 25: 405-416. 